\title{
SYNCHROTRON WHITE BEAM TOPOGRAPHIC STUDIES OF GALLIUM ARSENIDE CRYSTALS
}

\author{
W. WIERZCHOWSKI \\ Institute of Electronic Materials Technology \\ Wólczyńska 133, 01-919 Warsaw, Poland
}

K. WIETESKA

Institute of Atomic Energy, 05-400 Otwock-Świerk, Poland

AND W. GRaefF

HASYLAB at DESY, Notkestraße 85, 22603 Hamburg, Germany

\begin{abstract}
A series of samples cut out from different types of gallium arsenide crystals with low dislocation density were studied by means of white beam synchrotron topography. The investigation was performed with transmission and back-reflection projection methods and transmission section method. Some of the topographs in transmission geometry provided a very high sensitivity suitable for revealing small precipitates. The transmission section images significantly differed depending on the wavelength and absorption. In some cases a distinct Pendellösung fringes and fine details of dislocation and precipitates images were observed. It was possible to reproduce the character of these images by means of numerical simulation based on integration of Takagi-Taupin equations. Due to more convenient choice of radiation, synchrotron back-reflection projection topography provided much better visibility of dislocations than analogous methods realized with conventional $\mathrm{X}$-ray sources.
\end{abstract}

PACS numbers: $61.10 .-\mathrm{i}$

\section{Introduction}

$\mathrm{X}$-ray diffraction topography is widely used for characterization of gallium arsenide crystals and these investigations can also be performed with the use of synchrotron sources of $\mathrm{X}$-ray radiation [1-3]. It was recently shown [4, 5] that using more sensitive reflections of shorter wavelength and high indices results in improved detection of small inclusions and other microdefects. This may be easily realized with the use of synchrotron radiation providing a free choice of radiation wavelength and reflections and a better spatial resolution. 
In the present paper a series of samples cut out from different types of gallium arsenide crystals with a concentration of dislocations reduced by high doping was studied. The examination was performed with synchrotron transmission section and projection topography including the use of high sensitivity reflections. The possibilities of revealing dislocations in synchrotron back reflection topographs were also examined.

\section{Experimental}

The investigated samples were prepared from different types of gallium arsenide crystals grown both with Czochralski and horizontal gradient freeze (HGF) methods. Most of the crystals were obtained in the Institute of Electronic Materials Technology and kindly provided us by Dr. A. Hruban. The relatively low dislocation density on the level $10^{3} / \mathrm{cm}^{2}$ was obtained by doping with indium and silicon. Doping with indium was isoelectronic, i.e. by substitution of gallium atoms. In this case no additional impurity carriers are introduced. The indium concentration was $1-2 \%$, while the greatest concentration of silicon was $10^{18} \mathrm{~cm}^{-3}$. The samples were cut along (001) planes, perpendicular to the growth direction and their thickness was close to $400 \mu \mathrm{m}$. The samples were studied with different methods of X-ray diffraction topography realized using the white beam from the synchrotron DORIS III at DESY operating at $4.45 \mathrm{GeV}$.

Transmission synchrotron topography was used for the comparative studies of the results obtained in different samples. The topographs were recorded with the beam perpendicular to the sample. A single exposure produced a number of spots in different asymmetric reflections. The reflections were indexed using a numerical program. The projection topographs were taken with a relatively small beam size $8 \mathrm{~mm}$ horizontal $\times 2 \mathrm{~mm}$ vertical. After taking the projection topograph a horizontal slit limiting the beam front to $5 \mu \mathrm{m}$ was introduced in the middle of the beam and the section topograph was recorded in the same geometry. The investigation of larger areas were performed by taking successive topographs after translation of the crystal with respect to the beam.

The purpose of the white beam back-reflection projection experiments was to study the visibility of dislocations and other defects. In the present experiment the beam entered the crystal at an angle close to $15^{\circ}$.

\section{Results and discussion}

Part of the spots obtained on each exposure in transmission experiments corresponded to reflections of very short wavelength and high indices. In these reflections the contrast on defects becomes of extinction character, similarly as in conventional topography of low absorbing crystals built of elements with low atomic number. The sensitivity of the method seems to be greater than the sensitivity of Lang topography realized with Mo $K_{\alpha_{1}}$ and $\mathrm{Ag} K_{\alpha_{1}}$ in silicon crystal. In particular, the images of the dislocation lines are much broader than in the latter case. It may be also noticed that the images of many dislocations have the double contrast, as it was shown for many low absorbing crystal by Authier [6].

The effect of the increased sensitivity is in particular the visibility of small inclusions. The images of dislocations and point-like inclusions in highly silicon 
doped HGF-grown sample are illustrated in Fig. 1. Here the inclusions form a characteristic contrast built of two shallows. Most of these inclusions were invisible under the conditions most frequently used in conventional topographic investigations. Figure $1 \mathrm{~b}$ presents the images of dislocations in gallium arsenide highly doped with indium and small concentration of silicon. In this case we do not see the inclusions of point-like character. They decorate the dislocation lines and form a characteristic trail behind some of them.
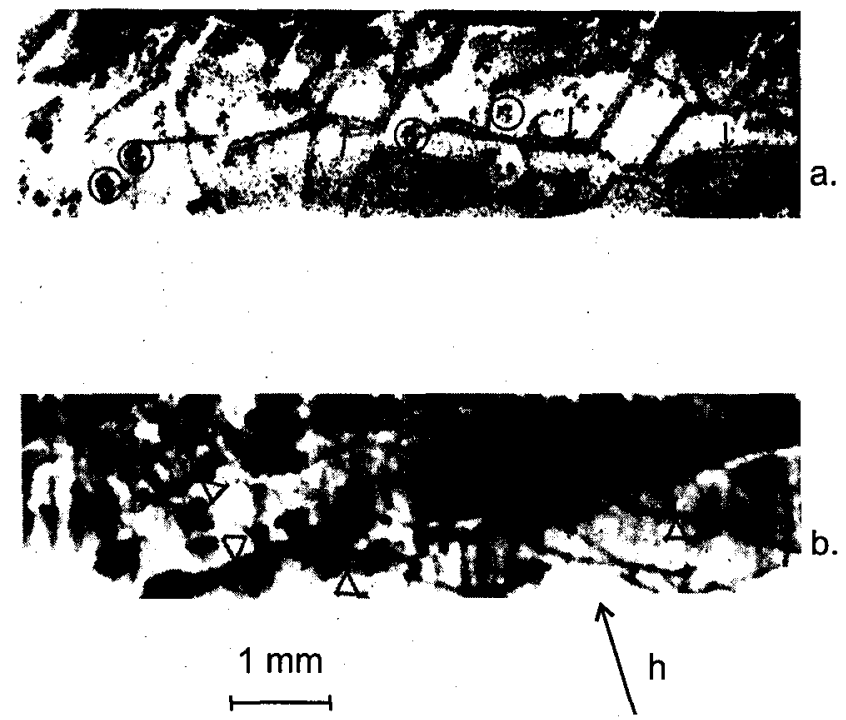

Fig. 1. Representative synchrotron transmission projection topographs of gallium arsenide samples in 1113 reflection of $0.25 \AA$ wavelength. The projection of the diffraction vector in all topographs is denoted by $h$. (a) Sample cut from heavily silicon doped crystal - the representative images of inclusions are marked by circles, and the dislocations with double contrast by arrows. (b) Sample from crystal double doped with indium and low addition of silicon. The characteristic trails, marked by triangles, are seen close to some of the dislocations.

Some interesting possibilities were found in the case of transmission section topography, which seems to be more sensitive method than projection topography. It was confirmed experimentally that in the case of low absorbed short wavelength, the best crystals of gallium arsenide can produce Pendellösung fringes. The best fringes were obtained in the sample cut from silicon doped Czochralski-grown crystal manufactured by the British firm MCP and they are shown in Fig. 2a. The crystal contained relatively low dislocation density but the projection topographs in sensitive reflections revealed some inhomogeneities in the dislocation-free regions. The fringes were also observed in some crystals grown in the Institute of Electronic Materials, in particular those grown with the HGF method. Figure $2 b$ shows a numerical simulation of dislocation images and the fringe system which match the experimental image. 


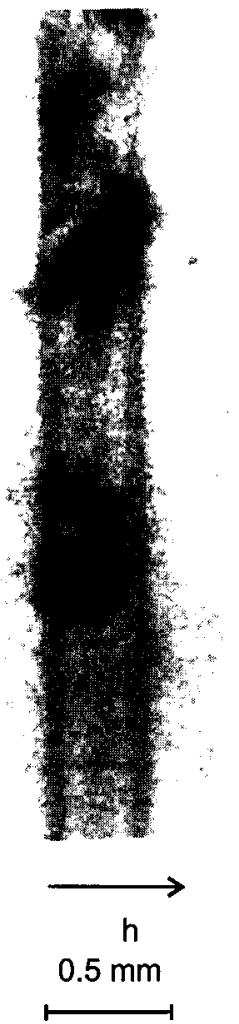

(a)

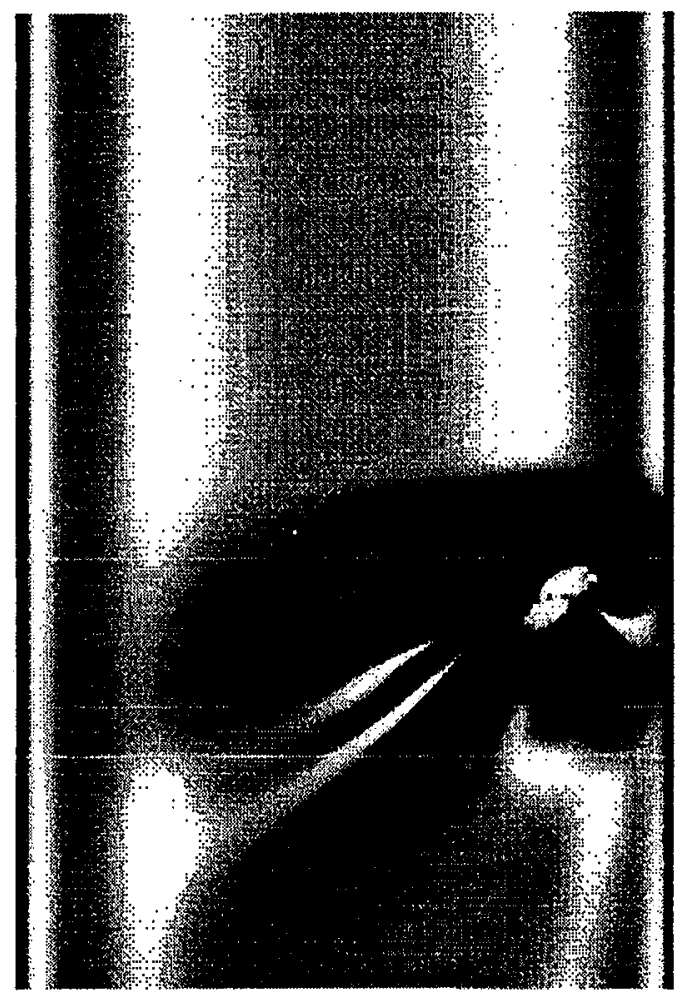

(b)

Fig. 2. (a) Pendellösung fringes obtained in silicon doped, Czochralski-grown gallium arsenide crystals in 773 reflection of $0.3 \AA$; (b) the simulated topograph of the dislocation image.
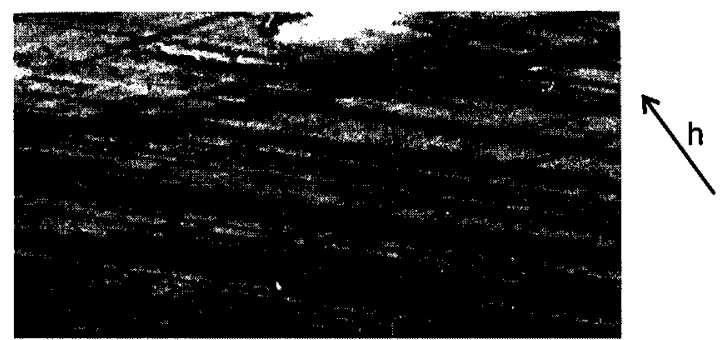

\section{$1 \mathrm{~mm}$}

Fig. 3. The back-reflection projection topographs in 535 skew reflection of $0.3 \AA$ revealing the misfit dislocations in homoepitaxial gallium arsenide layer on heavily indium doped substrate. 
White beam back-reflection projection topography, similarly as a BergBarrett and Lang back-reflection method is much less sensitive than the transmission one. In conventional $\mathrm{Cu} K_{\alpha_{1}}$ radiation the visibility of individual dislocations is not obvious [7]. Here the use of synchrotron white beam provides the possibility of improving the sensitivity and spatial resolution. The synchrotron pattern provides a number of skew reflections for which the planes of diffraction are not perpendicular to the surface of the sample. These reflections often select shorter wavelength and produce higher contrast on defects. Figure 3 presents the images of misfit dislocations in gallium arsenide homoepitaxial layer revealed using the skew 535 reflection selecting the wavelength $0.3 \AA$. Part of the contrast in Fig. 3 seems to be connected with growth bands and scratches.

\section{References}

[1] E. Prieur, T. Tuomi, J. Partanen, E. Yli-Jutt, M. Tilly, J. Cryst. Growth 132, 599 (1993).

[2] B.K. Tanner, D.K. Bowen, J. Cryst. Growth 126, 1 (1993).

[3] M. Surowiec, H.S. Leipner, J. Schreiber, J. Appl. Cryst. 22, 606 (1989).

[1] R. Barrett, J. Baruchel, J. Härtwig, F. Zontone, J. Phys. D 28, A250 (1995).

[5] J. Auleytner, V.I. Khrupa, L.I. Datsenko, S.M. Krasyulya, M.J. Skorokhod, Acta Phys. Pol. A 89, 301 (1996).

[6] A. Authier, $A d v$. $X$-ray Analysis 10, 9 (1966).

[7] S. O'Hara, M.A.G. Halliwell, J.B. Childs, J. Appl. Cryst. 5, 401 (1972). 